\title{
La línea de tres puntos y su influencia sobre la dinámica de juego en minibasket, el grado de satisfacción individual y competencia percibida de niños y nińas de la Región de Murcia
}

\author{
The three-point line and its influence on the gameplay in \\ Minibasketball, the degree of individual satisfaction and \\ perceived competence of children in the Region of Murcia.
}

\author{
María Teresa Mateo Mayol${ }^{1}$, María Teresa Miranda León² y David Cárdenas Vélez
}

${ }^{1}$ Facultad de Ciencias del Deporte. Universidad de Granada (España), ${ }^{2}$ Facultad de Medicina. Universidad de Granada (España).

\begin{abstract}
Resumen: El presente estudio de investigación se circunscribe a la competición de minibasket de la Comunidad Autónoma de la Región de Murcia, en la cual las reglas de juego establecen la presencia de una línea de tres puntos con unas características (forma y distancia) singulares. El objetivo fue comprobar la influencia de la modificación de la línea de tres puntos sobre el comportamiento individual y colectivo, así como el grado de satisfacción y competencia percibida de 43 jugadores (39 niños y 4 niñas) de cuatro equipos de minibasket inscritos en categoría alevín federada con una media de edad de casi 11 años. Para ello se organizó un torneo y se estableció un periodo de familiarización de dos semanas entre los dos días de competición. El juego de ataque experimentó cambios entre los que destacan, la ocupación más uniforme de los jugadores por el espacio, el incremento del tiempo de posesión del balón, un aumento proporcional del número de pases; los ataques adquirieron mayor profundidad y se realizaron más lanzamientos de tres puntos. Igualmente, se detectó un leve incremento del nivel de disfrute y el grado de competencia percibida. En conjunto, los datos muestran la conveniencia de una modificación de la regla referida a la línea de 3 puntos para favorecer un proceso de formación más adecuado a las características de la población objeto de estudio.

Palabras clave: adaptación, triple, lanzamiento, espacio, ataque.
\end{abstract}

Abstract: This research is limited to the minibasket competition of the Autonomous Community of the Region of Murcia, in which the rules establish the presence of a three-point line with specific characteristics (shape and distance). The objective was to test the influence of the modification of the three-point line on individual and collective behavior, and the degree of satisfaction and perceived competence of 43 players ( 39 boys and 4 girls) of four teams registered minibasket in juvenile category federated with a mean age of about 11 years. For this tournament it was organized and familiarization period of two weeks between the two days of competition was established. The attack game underwent changes among which, more uniform occupation of the players space, time increment possession, a proportional increase in the number of passes; the attacks became more depth and hit three points were made. Similarly, a slight increase in the level of enjoyment and the degree of perceived competence was detected.

Overall the data show the desirability of changing the 3-point line rule to promote a training process better fitted to the characteristics of children. Keywords: adaptation, three-point shot, shoot, space, offence.

\section{Introducción}

El minibasket es un buen ejemplo del intento de adaptar el deporte a las características de la población infantil (Lasierra y Lavega, 1993; Méndez, 1998). La edad y características de los participantes hacen recomendable una adaptación de las bases y reglas de competición para facilitar la consecución de los objetivos del juego y con ello incrementar la motivación. Igualmente, hay autores que consideran que las reglas debieran favorecer el descubrimiento y, por tanto, estimular por un lado el desarrollo de los mecanismos necesarios para la toma de decisiones y por otro, el aprendizaje de habilidades motrices transferibles a diferentes contenidos, garantizando así

Dirección para correspondencia [Correspodence address]: María Teresa Mateo Mayol. Facultad de Ciencias del Deporte. Universidad de Granada (Espańa).E-mail: mteresa_9173@msn.com la adecuada evolución cognitivo-motriz de los nińos (Graça y Oliveira, 1997). Este carácter pedagógico del minibasket queda reflejado en el conjunto de propuestas que aporta su reglamento, el cual configura la estructura formal y funcional del juego deportivo (Parlebas, 2001), y persigue una enseñanza lo más educativa posible (Piñar, 2005).

De hecho, el reglamento determina todas las condiciones necesarias para poder jugar y, en consecuencia, configura la lógica interna y la dinámica del juego (Hernández, 1998; Parlebas, 2001; Navarro, 2002; Lagardera y Lavega, 2003). Tal y como indican Cárdenas (2003), Ortega (2006) y Piñar (2005), es el elemento clave para lograr que la competición se convierta en un medio con valor formativo.

Semejante afirmación implica que la variación de algunas de las reglas puede ocasionar cambios estructurales y funcio- 
nales importantes que condicionarían la participación de los jugadores y el desarrollo táctico-técnico (Piéron, 1982; Silverman, 1985, 1990; Rink, 1993, 1996; Beaudet y Grube, 2005; Cárdenas, 2007). Esto induce a pensar que las modificaciones deberían realizarse con una doble perspectiva: asegurar que se conserven las características esenciales del juego (Mehl y Davis, 1978; Holt, Strean y García, 2002; Graça, 2006) y garantizar un mayor volumen de práctica de los contenidos de aprendizaje (Metzler, 2000). Considerando esta perspectiva, se hace necesaria una reflexión profunda de las condiciones de práctica que se producen en la competición actual de minibasket.

Las modificaciones de las reglas son posibles en los dos escenarios deportivos por excelencia: la competición y el entorno de práctica o entrenamiento. Mientras que el segundo tipo corresponde a los entrenadores, el primero depende de los estamentos federativos y su repercusión es mayor. En España, las modificaciones reglamentarias son responsabilidad de cada Comunidad Autónoma y de cada provincia y, desgraciadamente, suelen hacerse sin tener en cuenta la evidencia científica. Como problema ańadido, la Federación Espańola de Baloncesto no define claramente algunas de las reglas, por lo que las adaptaciones del reglamento de minibasket son, tal como afirma Cárdenas (2006), insuficientes y, en su defecto, deficientes. Sin embargo, conocer con certeza qué consecuencias provocarán tales modificaciones es una ardua tarea porque éstas dependen de la interpretación personal subjetiva que cada jugador hace de ellas (Coulter, 1971; Kew, 1990). Por otro lado, es relevante que muy pocos estudios han tenido como marco de referencia la competición. Entre todos, hemos considerado núcleo conceptual y procedimental de esta investigación el llevado a cabo por Piñar (2005), cuya publicación es la única que aborda la repercusión de jugar $3 \times 3$, en vez de $5 \times 5$. Además, esta analizó la incidencia de la modificación del espacio y tiempo de juego sobre la dinámica del juego.

En los últimos años se ha evidenciado un creciente interés por el análisis de la repercusión de la modificación de las reglas (Chase, Ewing, Lirgg, y George, 1994; Cárdenas, Piñar, y Baquero, 2001; Piñar, 2005; Ortega, Castro, y Laporta, 2008; Piñar, Cárdenas, Alarcón, Escobar y Torre, 2009). La amplia mayoría de estos trabajos se centraron en estudiar el efecto de las modificaciones del material o equipamiento deportivos, concretamente las dimensiones de la canasta y del balón. Por otro lado, un elevado número de este tipo de trabajos se realiza, en su mayor parte, en condiciones de laboratorio.

Algunos autores, pese a no presentar evidencias científicas que lo avalen, defienden propuestas de modificaciones que favorecen aspectos del juego recomendados en la iniciación al baloncesto, como la reducción del número de jugadores de 5X5 a 3X3 (Graça, 1998; Pinto, Graça e Ibáńez, 2002; Ca- rrillo y Rodríguez, 2004; Piñar, 2005). Este mismo cambio fue experimentalmente contrastado por Piñar (2005), junto con la utilización de una línea de tres puntos diseñada específicamente para los niños, la disminución del espacio de juego y la distribución equitativa del tiempo de juego para cada participante.

Teniendo en cuenta los estudios precedentes, entre los objetivos educativos que se plantean con la modificación o modificaciones reglamentaria/as probablemente el más importante sea incrementar las experiencias de éxito relacionadas con el contenido que, según consideran Cárdenas y Pintor (2001), entre otros (Rojas, Cepero, Oña y Gutiérrez, 2000), es el más importante y motivante de todos, el lanzamiento a canasta. Es por ello que la regla que define la línea de 3 puntos resulte de especial interés.

En esta línea de actuación, la Federación Española de Baloncesto (FEB) propuso para la temporada 2005/2006 unas modificaciones del reglamento de minibasket con el objetivo de conseguir una mayor y mejor adaptación del juego a las características de los niños. Entre las propuestas de cambio, la presencia novedosa de la incorporación de una línea de tres puntos se formuló con la intención expresa de que se realizaran más lanzamientos de larga distancia y que se incrementara el valor del cesto. Se conoce la existencia de ensayos realizados en varias federaciones autonómicas, como en el caso de la Canaria. No obstante, de éstos no se han encontrado, o no existen, ni propuestas ni resultados documentados.

La mayoría de expertos coinciden al afirmar que el diseño de la línea de tres puntos debe estar adaptado a las capacidades físicas, psicológicas y motrices de los jugadores (Arias, Argudo y Alonso, 2008). Para Ortega et al. (2004), la inclusión de la línea exterior de tres puntos permite estimular el desarrollo de la capacidad de lanzamiento de los jugadores, e incrementar el grado de variabilidad de este contenido. Además confiere cierta incertidumbre al ataque al diversificar las opciones de finalización. En definitiva, sus características deberían favorecer la aparición de situaciones de 1X1, el desarrollo de las habilidades motrices específicas y la capacidad para la toma de decisiones (Piñar, 2005). Sin embargo, autores como Mitjana se han mostrado reticentes a asumir la pertinencia de la línea de tres en minibasket (en el año 2007 consideró la línea que delimita el área de la zona restringida como línea de tres puntos). En cualquier caso, la decisión sobre su inclusión debe contemplar el análisis de la repercusión de las variables que la definen: forma y distancia a la proyección vertical del aro.

Por su parte, Arias et al. (2008) comprobaron la repercusión de dos ubicaciones diferentes de la línea de tres puntos sobre el número de situaciones de uno contra uno en minibasket femenino. Más concretamente, observaron una tendencia superior de situaciones de $1 \mathrm{X} 1$ jugando con la zona restringida, es decir, sin línea de tres puntos delimitada. Los 
datos resultantes fueron notablemente diferentes a los obtenidos por Piñar (2005) en minibasket femenino para una modalidad de $3 \mathrm{X} 3$ con línea de tres puntos y para una modalidad de $5 \mathrm{X} 5$ sin línea de tres puntos.

Tal como argumenta Piñar (2005), la inclusión de la línea de tres puntos a cuatro metros del aro provoca diferencias tanto a nivel colectivo como individual, entre las cuales destacan: el incremento del índice de participación, la disminución de su diferencia entre jugadores, así como el aumento del número de lanzamientos exteriores y de puntos conseguidos. Según la autora, esto supone para el nińo un aumento de su percepción de competencia y le proporciona una mayor posibilidad de satisfacer sus necesidades de éxito y de alcanzar, en última instancia, un elevado grado de satisfacción personal. Al igual que conseguir la adhesión a la práctica, requiere del niño o niña una buena actitud y pensamiento positivo hacia la tarea. Esto nos lleva a identificar una serie de factores o propiedades de tipo psicológico, entre los cuales destacan: la motivación, necesidad de logro, autoeficacia, autoestima y competencia motriz. El éxito en la iniciación debe ser entendido no como victoria sino como éxito personal fruto del esfuerzo empleado para asimilar la tarea (Roberts, 1991). Debido a esto, la competición debe estar diseñada de forma que se obtenga el éxito en un porcentaje elevado de intentos graduando su nivel de dificultad hasta llegar a conseguirlo de manera frecuente. Por esta razón en este estudio hemos analizado la posible repercusión de la modificación de la línea da 3 puntos sobre el nivel de disfrute y la competencia percibida.

Por otro lado, al estudiar el contexto particular de juego y las situaciones socio-motrices que se producen, se dirime que la lógica interna del baloncesto, condicionada por las reglas, establece como principio fundamental de acción (Alarcón, Cárdenas, Miranda y Ureña, 2010) la alternancia entre el juego exterior e interior. La ocupación y finalización mediante lanzamientos exteriores obliga a la defensa a extender su radio de acción, lo que genera a su vez mayor espacio interior disponible. Estas circunstancias llevan a los jugadores que desempeñan puestos específicos propios del juego exterior a ocupar posiciones alejadas de la canasta, pero desde la que resulten efectivos. Dada esta circunstancia, la línea de 3 puntos supone una referencia espacial determinante puesto que marca la distancia a partir de la cual el valor del enceste se incrementa de manera notable. Prolongando esta línea argumental, si la línea de tres puntos es semicircular los jugadores tenderán a situarse bordeándola por la parte más alejada de la canasta. Tal como pensamos, esta disposición inicial de ataque podría revertir en otros cambios comportamentales tanto individuales como colectivos. Dicho supuesto nos condujo finalmente a plantearlos la siguiente hipótesis inicial: La modificación de la linea de 3 puntos vigente en la Comunidad Autónoma de Murcia de forma rectangular por una linea semicircular a partir de los extremos de la línea de tiros libres y a la distancia de
4 metros de la proyección vertical del centro geométrico del aro, genera cambios en el comportamiento individual y colectivo de los jugadores de minibasket, así como en el grado de disfrute y competencia percibida.

El objetivo principal del presente estudio fue determinar la incidencia de las modificación reglamentaria (variable independiente) sobre los valores que presentan las acciones relacionadas con el juego colectivo y con el juego individual (variables dependientes), comparando el antes y el después (medida pre-post). Para ello tomamos como referencia al jugador con balón y consideramos, al mismo tiempo, la percepción subjetiva del jugador -disfrute y competencia percibida- .

En resumen, en el presente estudio tratamos de analizar el problema de cómo afecta la modificación de la forma de la línea de tres puntos, (rectangular a semicircular), tanto al comportamiento motor, como al grado de disfrute y competencia percibida. Asimismo, nos preguntamos si estas modificaciones en general podrían contribuir a la mejora del proceso formativo de los niños y niñas que practican minibasket en la región autónoma de Murcia.

\section{Método}

\section{A) Muestra}

Participaron 43 jugadores con una edad comprendida entre 10 y 12 años ( $M=10.65$ años). Los participantes formaban parte de cuatro equipos de minibasket de la Comunidad Autónoma de la Región de Murcia (muestra de conveniencia).

\section{B) Diseño}

El diseño es cuasiexperimental mixto multigrupo con medidas pre-post (sin-con modificación), y sin grupo control, donde los conjuntos considerados son el grupo experimental (Perea-Milla, 1998; Hernández, Fernández y Baptista, 2000).

El tipo de muestreo seleccionado ha sido de tipo no probabilístico de carácter opinático (Sierra-Bravo, 1995) o deliberado (Buendía, Colás y Hernández, 1998). La selección de este tipo de muestreo viene condicionada por las características propias del objeto de estudio y el acceso a la población participante.

\section{C) Materiales}

Los datos registrados se almacenaron mediante hojas de cálculo Excel 2012 de Microsoft (archivos de extensión .xls), y los partidos fueron filmados con cámaras de video Sony, modelo no: DRC-SR37E (1484238) y modelo no: DCR-SR32E (596656). Se utilizó una observación indirecta mediante análisis de vídeo con registro cuantitativo y continuo. Ello nos permitió una mayor fiabilidad en los registros (Lasierra, 
1993).

Tras la selección de observadores y fruto del proceso de instrucción de los mismos, similar al seguido por Piñar (2005), se elaboró un manual de instrucciones para ellos que constaba de: el sistema de categorías, la codificación de estas y el proceso de registro mediante el instrumento creado con este fin.

\section{D) Procedimiento}

La formación de los 4 observadores (voluntarios, estudiantes de la asignatura de Baloncesto de $3^{\text {er }}$ curso de la Facultad de Ciencias del Deporte de la Universidad de Granada), siguió las fases de entrenamiento y adiestramiento indicadas por Anguera y Blanco (2003). Este proceso se produjo durante seis sesiones, distribuidas en 15 días. Una vez finalizada la instrucción se obtuvo la fiabilidad del registro a través de la evaluación del rendimiento en relación con el de un observador de referencia. Para medir el índice de fiabilidad se llevo a efecto un estudio de confiabilidad entre los posibles observadores que consistió en una prueba control en situación de observación real, para lo que se seleccionó al azar un periodo de un partido de competición de cada una de las modalidades de juego. Para calcular el grado de fiabilidad, se utilizó el Coeficiente de Correlación Intraclase para variables continuas (cuantitativas) y el índice de concordancia para las variables categóricas (cualitativas) entre las transcripciones de cada uno de los observadores y el observador de referencia. Este cálculo se realizó mediante el coeficiente de concordancia Kappa, alcanzándose un valor superior a 0.95. Este valor es considerado como de "un acuerdo casi perfecto" (Altman, 1991). Finalmente, la toma de daros se realizó mediante un registro sistemático de observación activa, no participante y directa (Anguera y Blanco, 2003).

Para la configuración de la muestra se fijaron dos criterios de inclusión: a) que en los días de campeonato se debía jugar con los mismos jugadores y b) que en el tiempo que transcurriera desde el primer día hasta el segundo los entrenadores se comprometieran a seguir entrenando de forma habitual pero con la línea de tres puntos propuesta. Antes de proceder a realizar la parte empírica del trabajo se informó del propósito de la investigación a los responsables de los equipos y se obtuvo su visto bueno. Posteriormente, los entrenadores se encargaron de transmitir el motivo del torneo a los implicados y de recoger el consentimiento informado de los tutores legales, siguiendo la declaración de Helsinki.

Los equipos participaron en un torneo a doble vuelta, en el que los emparejamientos para competir entre los diferentes equipos se repetían en dos fines de semana consecutivos. En el primer día de torneo se jugó con las reglas habituales (medida pre: figura 1) y en la segunda jornada, siete días después (medida post), se jugó con la modificación de la línea de tres puntos (figura 2). Desde la temporada 2005-2006 en la competición federada de la Comunidad Autónoma de la Región de Murcia de minibasket la línea de tres puntos está situada a 4 metros del aro, al igual que la distancia del tiro libre, y tiene forma rectangular (Figura 1 y 2).

Figura 1. Formato de campo con la líneas de tres puntos rectangular (izquierda) y semicircular (derecha).

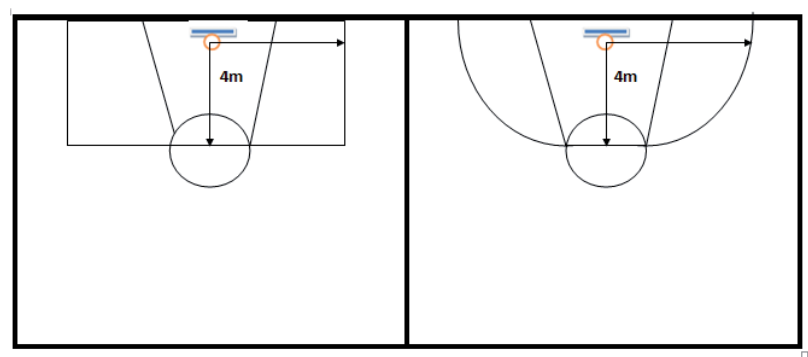

Figura 2. Resultado de trazar la línea de tres puntos semicircular en una mitad del campo de minibasket.

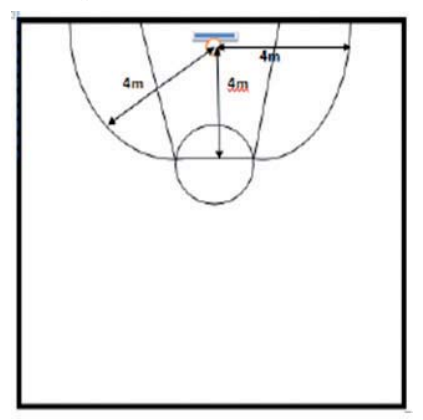

La modificación supone cambiar la línea rectangular por una semicircular, trazada a 4 metros de la proyección vertical del centro del aro. Esto supone que la distancia permanece invariable.

Variables motrices:

Variables relacionadas con el juego colectivo: Número de Fases de Ataque (Fat), Duración de las Fases de Ataque (Dfa), Tipo de Ataque (TAt), Número de Pases (Np).

\section{Número de Fases de Ataque (Fat)}

Número de fases de ataque en las que el equipo analizado dispone del control o posesión del balón. Comienza cuando el equipo recupera la posesión del balón y finaliza cuando la pierde.

\section{Duración de las Fases de Ataque (Dfa).}

Se registra, en segundos, la duración de cada fase de ataque. En los estudios de Ortega, Piñar y Cárdenas (1999) y Ortega (2004) se establecieron cinco categorías. En el presente estudio se optó por reducirlas a tres y variar el rango de tiempo seleccionado para cada una de ellas: una categoría que aglutinara las fases de ataque con una duración superior a 15" y dos con un rango de segundos similar (7-8”). 
2.1. De 0 a 8 segundos; 2.2. De 9 a 15 segundos; 2.3. Más de 15 segundos

3. Tipo de Ataque (TAt).

Se registra el tipo de fase de ataque que un equipo lleva a cabo cuando obtiene la posesión del balón, estableciendo tres categorías:

3.1. Contraataque (1). Transición de la defensa al ataque que se produce con la mayor velocidad posible para conseguir superioridad numérica, ventaja posicional o ventaja táctica. En el presente estudio se han analizado los contraataques en primera oleada. Se entiende por primera oleada del contraataque a una primera fase de la transición en la que intervienen los jugadores que se incorporan en primer lugar y que, normalmente, son los que se encuentran más cerca de la pista delantera en el momento de la recuperación del balón.

3.2. Ataque posicional (2). Fase del juego que discurre a partir del transporte del balón a pista delantera, una vez que la defensa rival se encuentra correctamente posicionada para evitar la aproximación hacia la canasta cómoda y rápida, tanto del balón como de los jugadores. Se entiende que es el tipo de ataque que se produce contra una defensa organizada. La continuación del ataque tras la captura de un rebote ofensivo forma parte del mismo ataque posicional. En este caso, tras la recuperación del balón continúa la acción de ataque.

3.3. Otros (3). La fase de ataque comienza e inmediatamente finaliza por una pérdida del balón, una falta personal, una interceptación de balón... (Ej. Un jugador saca de fondo para pasar a un compañero y cuando éste recibe pierde el balón porque se lo roban, comete una violación, recibe una falta personal, etc.).

4. Número de Pases (Np). Número de veces que los miembros de un equipo se pasan el balón. Se establecen tres categorías (Piñar, 2005): 4.1. De 0-5 pases; 4.2. De 6-10 pases; 4.3. Más de 10 pases.

Variables relacionadas con el juego individual del jugador con balón: Posiciones por donde se desplaza el jugador con balón (Ped), Finalización de la Posesión del balón (FinP), Posiciones de Lanzamiento (Pel) y Distancias de Lanzamiento (DistL), Periodos de Juego (P), Uno Contra Uno Jugados (1x1), Rendimiento del Jugador (Rend) medidos a través de los puntos en juego conseguidos (2 ó 3 puntos), Puntos de Tiro Libre Convertidos (TL) e Intentos de Lanzamiento en Juego (IntL). (Ped).

5. Posiciones por donde se desplaza el jugador con balón

Se registran los diferentes espacios utilizados por el jugador cuando se desplaza con el balón en las manos, modificando la propuesta de Pintor (1987). A diferencia de Piñar
(2005), se ha considerado sólo la categoría de posiciones específicas de desplazamiento (Ped) (Figura 3).

Figura 3. Posiciones específicas de desplazamientos y lanzamientos. 1: Base (B); 2: Alero Alto (AA); 3: Alero Medio-Bajo (AMB); 4: Pívot Derecho (PD); 5: Pívot Izquierdo (PI); 6: Pívot Bajo (PB); 7: Pívot Alto (PA).

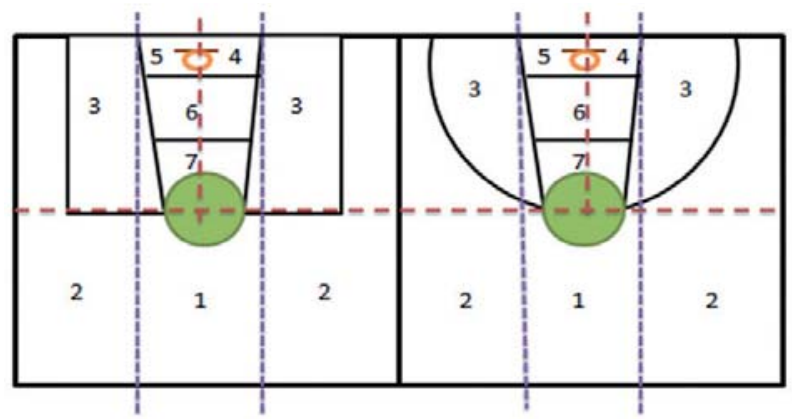

Figura 4. Distancias de desplazamientos y lanzamientos (DistL). P: Próxima (periferia del aro); C: Corta (dentro del área restringida); M: Media (dentro de la zona de tres puntos); L: Larga (fuera de la zona de tres puntos); E: Extrema (más allá de la línea central del campo).

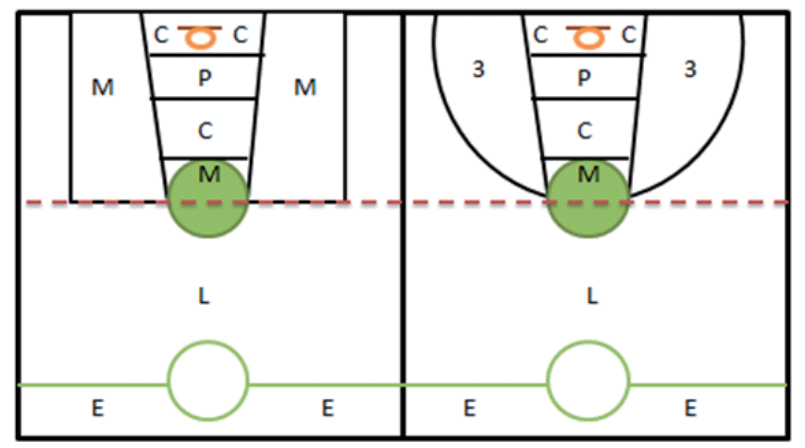

6. Finalización de la Posesión del balón (FinP). Se registra la forma en que un jugador deja de tener el control del balón.

6.1. Pase (P). El jugador pierde la posesión del balón al realizar un pase a un compañero.

6.2. Acciones en contra (Ac). Se registran aquellas acciones que no favorecen al equipo que controla el balón y que pueden ser realizadas por cualquier jugador (pérdida de balón, falta personal, técnica o descalificante, 3 seg. en zona y campo atrás).

6.3. Acciones a favor (Af). Se registran aquellas acciones que favorecen al equipo que controla el balón, realizadas o no por el jugador con balón (recuperaciones, falta personal recibida, taponar, capturar un rebote,...).

7. Posiciones y Distancias desde donde Lanza el jugador con balón. Se registran los diferentes espacios desde donde el 
jugador con balón realiza el lanzamiento. Se consideran dos categorías: posiciones específicas de lanzamiento (Pel) y distancias de lanzamiento (DistL), excepto la distancia próxima trasera.

8. Periodos de Juego (P). Se registra el número de periodos disputados por cada jugador.

9. Uno Contra Uno jugados ( $1 x 1)$. Se registra el número de $1 x 1$ que realiza el jugador con balón. Para el análisis de esta categoría se ha considerado $1 x 1$ a toda situación de confrontación directa con el adversario, que se produzca en pista delantera, durante el transcurso del ataque posicional y en la que se aprecie intención de dirigirse hacia la canasta contraria para conseguir encestar aunque no se produzca finalmente el lanzamiento. No se contempla $1 \times 1$ en la situación de contraataque.

10. Rendimiento del Jugador (Rend). Se registra el número de puntos conseguidos por cada jugador, pudiendo ser 0 puntos, 1 punto, 2 puntos, 3 puntos, o más de 3 puntos. En el caso de producirse falta personal con lanzamiento/s de tiro/s libre/s, se estipula que el rendimiento de la fase de ataque es igual al número de tiros libres encestados más el valor del enceste si se hubiera producido. Se analizan por separado.

10.1. Puntos en juego conseguidos. Se diferencia entre: 10.1.1. Lanzamientos convertidos de 2 puntos.

10.1.2. Lanzamientos convertidos de 3 puntos.

10.2. Puntos de Tiro Libre convertidos (TL).

10.3. Intentos de Lanzamiento en juego. Número de veces que un jugador realiza un lanzamiento en situación de juego (excluyendo el lanzamiento desde la línea de tiro libre). Se distinguen: 10.3.1. Intentos de dos puntos (Int2). Lanzamientos que se realizan desde una distancia $\leq 4 \mathrm{~m}$.; 10.3.2. Intentos de tres puntos (Int3). Lanzamientos que se realizan desde una distancia $>4 \mathrm{~m}$.

Variables Psicológicas:

En el presente estudio también se analizó una variable de tipo cualitativo, la percepción subjetiva de disfrute y competencia percibida por los niños. Para ello se utilizó el cuestionario BECS (Arias, Alonso y Yuste, 2012). El cuestionario consta de 7 preguntas simples en las que hay que señalar el grado de acuerdo en una escala con valores que oscilan entre el 1 y el 5 , en orden ascendente.

\section{E) Análisis estadístico}

Para todo el análisis estadístico se utilizó el Paquete de programas estadístico SPSS v.20 (SPSS Inc. Chicago, IL, EEUU). El análisis de los datos se realizó considerando que se trataba de variables categóricas o categorizadas. Los procedimientos estadísticos descriptivos fueron básicamente la elaboración de Tablas de frecuencias y porcentajes para cada una de las variables así como su representación gráfica por medio de diagramas de barras y en cuanto a los métodos de inferencia la elaboración de tablas de contingencia. En estas el test aplicado fue, bien el test Chi-Cuadrado de Pearson si se cumplían las condiciones de validez (ninguna frecuencia esperada puede ser menor que uno y no más del 20\% de las casillas pueden tener frecuencias esperadas menores o iguales que 5) o el Estadístico Exacto de Fisher, para el caso en que no se cumplieran dichas condiciones. Para las variables cuantitativas se realizó el test de comparación de medias t-Student o Welch o test no paramétrico de Mann-Whitney o Wilcoxon en caso de variables no normales.

Para todas las comparaciones se tomó como nivel mínimo de significación el valor $\mathrm{p}<0,05$, considerándose un resultado muy significativo si $\mathrm{p}<0,01$ o altamente significativo si $\mathrm{p}<0,001$.

\section{Resultados}

De todas las variables descritas y estudiadas, sólo se reportan los datos de aquellas que han resultado más significativas y relevantes para el objetivo general del estudio. En última instancia las variables que han resultado excluidas son: los Periodos de Juego (P) y el Rendimiento del Jugador (Rend).

En relación con el juego colectivo:

La línea de tres puntos semicircular a 4 metros de distancia provocó un aumento de la duración de las fases de ataque en más de 10 s., lo que representa un incremento del 50,1\%, $(\mathrm{p}<0.001)$, tal y como se puede observar en la Figura 5. Tanto con la línea rectangular como semicircular, el ataque posicional predominó sobre los demás, representando el 69,5\% de las acciones ofensivas. No obstante, se observó mayor variabilidad en el tipo de ataque (contraataque, ataque posicional y otros) al jugar con la modificación de la línea, es decir, con la nueva línea de 3 puntos aumentó sutilmente el número total de tipos de ataques $(50,1 \% ; \mathrm{p}<0.001)$. A este respecto, cabe destacar el incremento de los ataques definidos como Otros 1 $(9,3 \%$ sin la modificación y $11,7 \%$ con ella; $\mathrm{p}<0.001)$.

1 Otros: posesiones que rápidamente terminan con una pérdida del balón (acción en contra o robo).

Efectos de un programa de actividad física intensa en la composición corporal de... SPORT TK, 5(2), 89-102 
Figura 5. Porcentaje de Fases de Ataque con un duración superior a 15 segundos por modalidad y partido, respecto al conjunto total de partidos, $(\mathrm{p} \leq 0.05)$. Los equipos están indicados con las letras A, B, C y D.

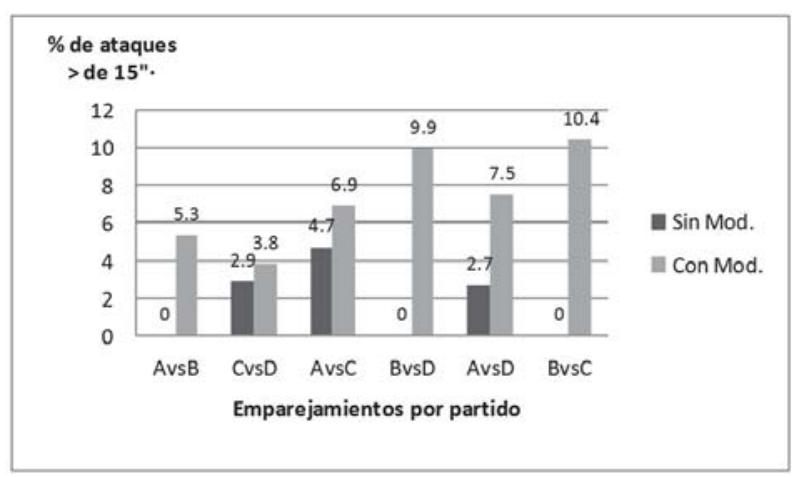

Además, el número de pases por fase de ataque aumentó considerablemente. La modificación de la línea provocó un aumento del número de fases de ataque $(\mathrm{p}<0.001)$ en las que se produjeron de 6 a 10 pases (61,7\% con la modificación). Este hecho causó en todos los partidos disputados el descenso proporcional de los ataques en los que se efectuaban un número de pases inferior (entre $0-5$ pases; 49,2\% con la modificación), lo que supone un incremento de la participación de los jugadores en las fases de ataque $(\mathrm{p}<0.001)$.

Figura 6. Número de fases de ataque por partido en las que se realizaron entre 6-10 pases $(\mathrm{p}<0.001)$.

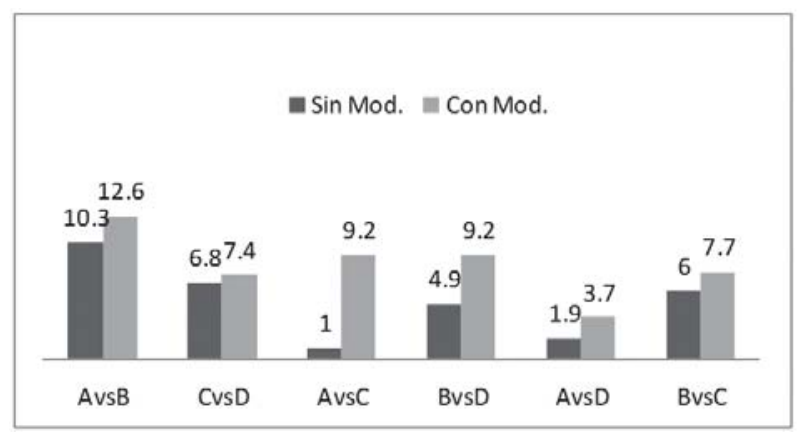

De forma más específica, se observa que jugando con la modificación los equipos A y $\mathrm{C}$ incrementan el número de fases de ataque en las que se realizaron 6 o más pases $(61,7 \%$ frente al 38,3\%). Además, con la línea rectangular ningún equipo, salvo el $\mathrm{C}$, realizó más de 10 pases por fase de ataque. Al margen de la heterogeneidad de los resultados, diferenciando por equipos, en la categoría de 6-10 pases el resultado global muestra diferencias con indicios de significación $(\mathrm{p}=0.057)$.
Figura 7. Porcentaje de fases de ataque por equipo con una frecuencia de 6-10 pases.

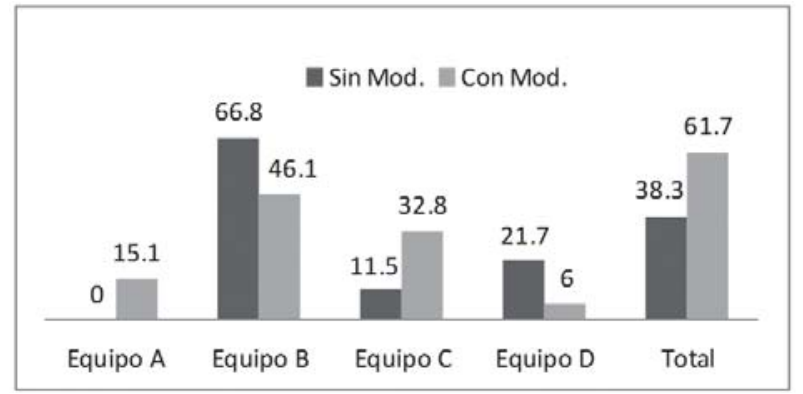

Asimismo, se realizaron más pases exteriores $(52,3 \%$ con la modificación y $47,7 \%$ sin la modificación; $\mathrm{p}<0.001$ ), aunque sólo en 3 de los 6 enfrentamientos las diferencias fueron estadísticamente significativas $(\mathrm{p} \leq 0.05)$.

\section{En relación con el juego individual del jugador con balón:}

Con relación a la ocupación individual espacial, descartando la posición de base que es por la que más transcurre el balón, se observa una alta ocupación de los espacios laterales (alero alto y alero medio-bajo). Concretamente, se detectó una mayor ocupación de las posiciones de alero alto y bajo, y la de pívot bajo, jugando con la línea semicircular (Véase la Figura 8).

Figura 8. Posiciones Específicas de Desplazamiento más ocupadas con la modificación de la línea de 4 metros.

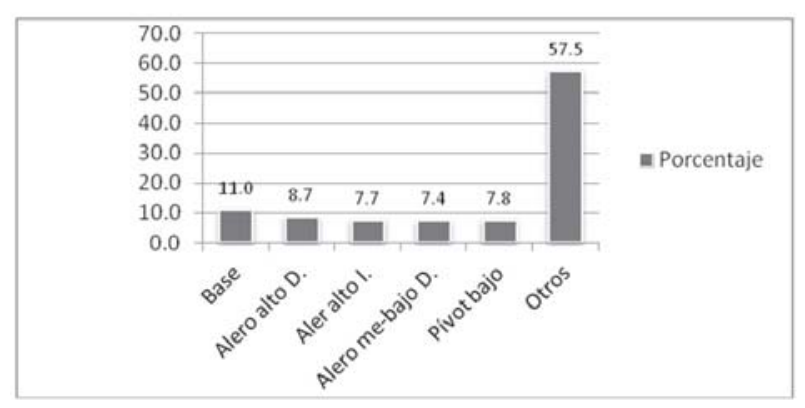

La categoría denominada como Otros aglutina las posiciones con porcentajes más bajos e intrascendentes, es la suma de todos estos porcentajes individuales restantes.

Igualmente, el análisis global muestra un aumento del número de lanzamientos desde la línea de tres puntos el segundo día de competición $(52,5 \%$ de la media de todos los partidos jugando con la línea semicircular frente al 47,5\% sin la modificación) sin llegar a alcanzar niveles mínimos de significación estadística $(\mathrm{p}=0.927)$. Sin embargo, en 5 partidos se hallaron diferencias altamente significativas $(\mathrm{p} \leq 0.05)$, así como un leve aumento del porcentaje de acierto $(60,9 \%$ 
con la modificación). Por el contrario, no se apreciaron diferencias estadísticamente significativas en el número de tiros libres encestados $(\mathrm{p}>0.05)$.

Figura 9. Número de lanzamientos de tres puntos $\left({ }^{*} \mathrm{p} \leq 0.05\right.$ valores expresados en $\%$ del total por partido)

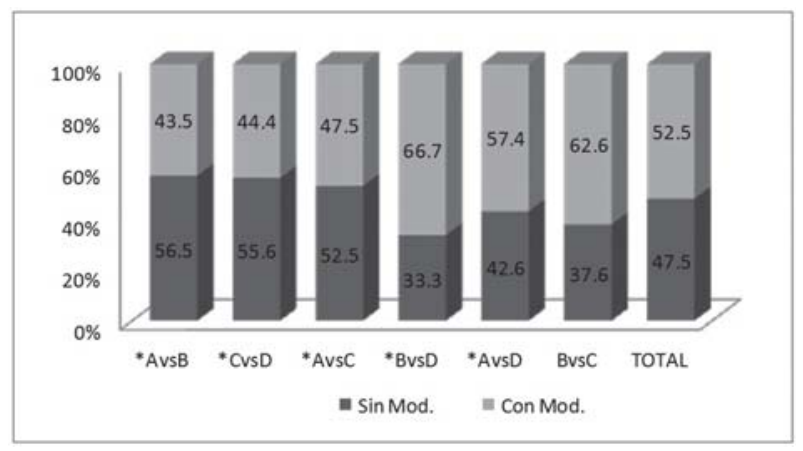

Con la nueva línea de tres puntos se ejecutaron más lanzamientos exteriores por partido (Figura 9) y menos interiores $(48,5 \%$ con la modificación; $\mathrm{p} \leq 0.05)$.

La escasa variabilidad en el número de $1 \mathrm{X} 1$ jugados en función del tipo de línea de tres puntos utilizada (el 19,5\% de los ataques jugando sin la modificación frente al 16\% con la modificación) y la pluralidad de resultados para los pases interiores $(\mathrm{p} \geq 0.05)$, muestra leves modificaciones en el juego en profundidad asociadas al cambio reglamentario propuesto.

\section{En relación con las variables Psicológicas}

Respecto al grado de satisfacción personal y de competencia percibida (Tabla 1) los resultados no mostraron diferencias significativas como consecuencia de la modificación reglamentaria $(\mathrm{p} \geq 0.05)$.

Tabla 1. Sumatorio de las medias de todos los equipos de las variables de competencia percibida y satisfacción personal (disfrute); $\mathrm{p} \geq 0.05$.

\begin{tabular}{lll}
\hline DíA & Comp. Percibida & Disfrute \\
\hline 1 & $\sum \mathrm{NM}=3,92$ & $\sum \mathrm{NM}=4,87$ \\
2 & $\sum \mathrm{NM}=3,94$ & $\sum \mathrm{NM}=4,90$ \\
\hline
\end{tabular}

\section{Discusión}

El objetivo fundamental de este estudio era comprobar si la modificación de la forma de la línea de tres puntos podría modificar la dinámica del juego del minibasket para convertirlo en un medio formativo más eficaz. Entre los posibles cambios que contribuirían a mejorar las experiencias competitivas de los jugadores, los autores especializados en la ma- teria destacan el incremento del contacto individual con el balón (Piñar, 2005), del número de situaciones de 1X1, del número de lanzamientos y particularmente de lanzamientos exteriores (Arias, 2007; Arias, et al., 2008), así como de la variabilidad en las formas de participación motriz (Cárdenas, et al., 2001; Ortega, 2006; Carrillo, et al., 2009; Arias, et al., 2011).

Por otra parte, las modificaciones deberían ayudar a reforzar la autoestima de los jugadores y proporcionar experiencias gratificantes que aumenten su motivación por la práctica deportiva.

Nuestros resultados refuerzan parcialmente estos supuestos. Concretamente, muestran un aumento del número de veces que los jugadores participaron, como consecuencia de la realización de más pases por fase de ataque. Este hecho podría estar directamente relacionado con la mayor duración de las posesiones de balón.

Asimismo, los ataques fueron más variados, aunque con un claro predominio de los ataques posicionales. Este dato puede tener una doble interpretación: por un lado positiva al suponer un aumento de la variabilidad motriz colectiva y por otro negativa si representa una reducción del número de contraataques. No obstante, pese a no obtener datos específicos más concluyentes, este resultado nos conduce a pensar que el aumento del tipo de ataque categorizado como Otros es consecuencia de jugar con la nueva línea de 3 puntos, lo cual motivó nuevas formas de experimentación técnico-táctica en los participantes.

El juego en profundidad se manifiesta mediante diversos parámetros tácticos, entre los cuales se encuentra el $n^{\circ}$ de pases interiores, los lanzamientos próximos al aro, las situaciones de 1X1, y la ejecución de Tiros Libres. Al analizar la respuesta de los participantes en el empleo del 1X1 no se encontraron diferencias significativas al modificar la forma de la línea actual de tres puntos. Autores como Cárdenas y Piñar (2004) y Ortega, et al. (2004), destacan la necesidad de incluir una línea de tres puntos para favorecer el incremento de situaciones de 1X1. Sin embargo, el estudio de Piñar (2005) mostró un escaso número de situaciones de $1 \mathrm{X} 1$ jugadas tanto sin línea como al incluirla. En nuestro caso, la comparación se produce entre diferentes modalidades de líneas de tres puntos y no entre el hecho de jugar con o sin línea. En este sentido, Arias, Argudo y Alonso (2008) analizaron las situaciones de $1 \mathrm{X} 1$ jugadas por posesión del balón utilizando dos líneas de tres puntos: una coincidente con la que delimita el área restringida y otra de forma rectangular propuesta por la FEB en 2005. Llamativamente se observó una tendencia superior de situaciones de $1 \mathrm{X} 1$ jugando con una línea de tres puntos que coincidía con la que delimita el área restringida $(M=0.28, S D=0.48$ frente al $M=0.25, S D=0.50)$. Dado que la distancia al aro desde las zonas laterales de la $2^{\text {a }}$ línea es mayor que en la otra alternativa, parece que la falta de ali- 
ciente o refuerzo extrínseco para lanzar desde lejos provocara la tendencia a atacar con un mayor sentido de profundidad. En cualquier caso, atendiendo a las recomendaciones de los expertos en el ámbito de la formación deportiva (Piñar, 2005; Arias et al., 2008), el número de situaciones de $1 \mathrm{X} 1$ que registramos en ambos campeonatos fue reducido.

La escasa utilización del 1X1, como recurso frecuente del juego de ataque, puede deberse principalmente a las carencias técnico-tácticas de los jugadores de iniciación. No obstante, una posible explicación encuentra apoyo en la relación inversamente proporcional entre el número de penetraciones y el de lanzamientos a canasta. Nuestros datos muestran un incremento del número de lanzamientos exteriores que inevitablemente reflejan una reducción del número de penetraciones. Además, es importante considerar como limitación la propia definición de la situación de 1X1 utilizada, que considera como tal al conjunto de acciones que permiten al jugador aproximarse a canasta para finalizar el ataque. Esto excluye aquellas situaciones en las que el jugador comienza la acción de bote para generar un espacio exterior desde el que lanzar y que suponen acciones de arrancadas pero también de paradas. Es evidente que para el futuro convendría contemplar la diferenciación entre situaciones de 1X1 que finalicen con penetración y con tiro exterior.

Existen otras razones que podrían explicar los resultados pero no han sido experimentalmente contrastadas, por lo que las planteamos a modo de hipótesis para futuras investigaciones. Una de estas razones podría ser la tendencia defensiva de los equipos rivales, es decir, si los contrarios conceden una mayor distancia interpersonal, por una carencia en la efectividad del lanzamiento exterior, los atacantes verán mermadas sus posibilidades de penetrar. Por otra parte, si se producen penetraciones, una de las formas de finalización de la posesión individual más eficaces es el pase al compañero desmarcado siempre que la penetración haya provocado la fijación del oponente indirecto. En este caso, si el pase al jugador desmarcado es interior, el descenso de $1 \mathrm{X} 1$ podría acarrear también el descenso del $n^{\circ}$ de pases interiores. No obstante, tanto la poca realización del $1 \mathrm{X} 1$ como el consecuente desuso del juego interior, apenas afectó sobre el número de tiros libres encestados, viéndose poco o nada alterado.

Por otro lado, nuestros datos muestran que las posiciones más ocupadas por los desplazamientos fueron las de base, alero alto y medio-bajo derecho e izquierdo, y pívot bajo, sumando un total del $42,95 \%$ respecto a la cantidad global de desplazamientos. A la vista de los datos, podemos establecer cierta similitud con los resultados de Piñar (2005), quien obtuvo que un $33 \%$ de los desplazamientos en minibasket, en ataque y defensa, se realizaron por las posiciones de base, alero alto derecho y pívot alto, y a distancias que superaban los tres metros (66,7\% del total de los desplazamientos).

En cuanto a la participación de los jugadores, los datos muestran la realización de más pases jugando con la nueva línea a 4 metros $(52,3 \%$ con la línea de tres puntos semicircular). Además, se produjeron más lanzamientos, apreciándose incluso un incremento de los ejecutados a larga distancia $(52,5 \%$ con la línea de tres puntos semicircular frente al $47,5 \%$ jugando con la línea exterior actual; $\mathrm{p} \leq 0.05$ ).

El lanzamiento a canasta es la habilidad motriz específica que más se relaciona con la consecución del objetivo final del juego de minibasket. Esto implica que cuanto mayores sean las posibilidades del jugador de realizar lanzamientos a canasta, mayores serán también las de obtener éxito y por tanto de incrementar la seguridad en sí mismo (Roberts, 1991; Giménez y Sáenz-López, 1996, 1999; Cárdenas, 1999, 2004; Ortega et al., 1999; Cárdenas y Pintor, 2001; Torre et al., 2001; Piñar et al., 2002; Ortega et al., 2004). La investigación realizada hasta la fecha demuestra que la mayor parte de los lanzamientos en minibasket se realizan desde el interior de la zona restringida, (Piñar, Alarcón, Vegas, Carrero y Rodríguez, 2002; Piñar et al. 2003). Para este tipo de lanzamientos Piñar et al. (2002 y 2003) obtuvieron valores del 81\% y del $69,5 \%$ en ambos estudios respectivamente. Sin embargo, Piñar (2005) halló un 71,6\% para minibasket femenino. También encontró un aumento del 17\% en el número de canastas encestadas. No obstante, al haber incluido varias modificaciones no es posible establecer una clara relación causa-efecto y deducir que los resultados fueran sólo consecuencia de la inclusión de la línea de tres puntos. Coincidimos entonces en la necesidad de analizar cuál de los dos modelos de la línea de tres puntos puede favorecer y/o perjudicar la realización de las acciones motrices que deben predominar en minibasket. Tal como hemos comprobado, en el presente experimento, la modificación de la línea de tres puntos produjo más lanzamientos de larga distancia (Véase la Figura 9) y con mayor tasa de éxito, en 3 de los 6 partidos disputados.

Además, con la línea de tres puntos semicircular, al ejecutar el lanzamiento más allá de cuatro metros se ocuparon más los espacios laterales que los centrales. Consiguientemente, y a pesar de la invariabilidad del número de $1 \mathrm{X} 1$ registrados, hallamos una tendencia creciente en los lanzamientos lejanos a canasta desde posiciones laterales, esquinas y diagonal hacia el aro.

De acuerdo con autores como Arias (2008) o Cárdenas y Pintor (2001), el minibasket debe lograr que los jugadores participen acumulando el mayor número posible de contactos con el balón. La participación con balón de los jugadores en cada posesión, así como el número de lanzamientos a canasta desde las diferentes posiciones, favorecerá un mayor desarrollo de las habilidades motrices de los jugadores. No obstante, los antecedentes han demostrado que en minibasket la participación con balón se restringe a uno o dos jugadores, que son habitualmente los mismos (Pińar, 2005). Arias et al. (2008) observaron como a medida que aumentaba el 
número de jugadoras participantes, disminuía el porcentaje de posesiones en las que esto ocurría. En cambio, Piñar et al. (2009) señalan la necesidad de participar en una competición reducida y simplificada con el fin de ofrecer una participación activa y directa con el móvil, como requisito indispensable para la mejora de los contenidos del juego (ASEP, 2001; Piñar, Cárdenas, Conde, Alarcón y Torre, 2009).

El jugador, en el transcurso de la competición, tiene que contar con oportunidades de lanzar y de conseguir éxito al hacerlo, lo que aconseja crear las condiciones que lo faciliten (Cárdenas y Pintor, 2001; Mondoni, 2001), modificando y adaptando las reglas al jugador de minibasket (Weiss \& Gould, 1986; Roberts, 1991; Chase, Ewing, Lirgg \& George, 1994; Blázquez, 1995; Chalip \& Green, 1998; Giménez y Sáenz-López, 1999; Cárdenas et al., 2001; Cárdenas, 2003). Del mismo modo, Cárdenas y Pintor (2001) consideran que el enceste es experimentado por el nińo como un éxito que refuerza su autoestima y le ayuda a progresar como jugador. Podemos concluir pues, que las reglas dan sentido y un fin único al juego pues condicionan la dinámica y las acciones que se producen.

Conseguir la adhesión a la práctica requiere que el niño o nińa tenga una buena actitud y pensamiento positivo hacia la tarea, lo que depende de una serie de factores psicológicos, entre los cuales se encuentran la motivación, necesidad de logro, autoeficacia o sensación de competencia, autoestima y la propia competencia motriz. A este respecto cabe mencionar a Arias, Alonso y Yuste (2012), quienes midieron las propiedades psico-afectivas de disfrute y competencia percibida de forma adaptada al baloncesto de iniciación (BECS). Los participantes del estudio declararon un Disfrute $(M=4.44$; $D E=0.41)$ y una Competencia percibida $(M=4.49 ; D E=$ 0.41 ) de tendencia elevada, considerando un rango de valores mínimos y máximos de 1 a 5, respectivamente. Por otro lado, en el trabajo de Piñar, Cárdenas, Conde, Alarcón y Torre
(2007) se comprobó que el 90\% de los jugadores menores de 11 años encuestados se sentían bien, y con altos niveles de satisfacción, cuando participaban más en el juego de su equipo, tenían más posibilidades de realizar acciones y jugaban más tiempo durante la competición. Aunque en el presente estudio nuestros resultados fueron igualmente elevados en ambos días de competición, se observó un leve aumento el segundo día de torneo, lo cual podría favorecer la adherencia a la práctica deportiva (Navarro, 2002; Ortega, 2006; Carrillo, y Rodríguez, 2009; Casey, et al., 2010; García, et al., 2013). No obstante, hay que resaltar que dichas diferencias no fueron significativas.

El éxito en la iniciación debe ser entendido no como victoria sino como logro personal fruto del esfuerzo empleado para asimilar la tarea (Roberts, 1991). Es por ello que la competición debe estar diseñada de forma que se obtenga éxito en un porcentaje elevado de intentos, graduando su nivel de dificultad hasta conseguirlo de manera frecuente.

A pesar de que el baloncesto es uno de los deportes sobre el que existen más estudios que analicen el efecto de modificar las reglas para adecuarlo a las características de los niños, el volumen de trabajos de investigación que reportan argumentos válidos en los que fundamentarse, es realmente escaso. Por otra parte, los estudios contrastados muestran propuestas de modificación dispares y obtienen resultados contradictorios que hacen recomendable continuar investigando en este campo.

Agradecimientos.- A los clubes de baloncesto (CB Sierramar, CB Cartagena, EBS Salesianos, y CB Estudiantes Cartagena) jugadores, familiares y patrocinadores participantes en el estudio. Mención especial al Club Baloncesto CB Sierramar de el Algar (Cartagena), por su gran implicación en la organización y desarrollo del torneo creado para este fin, y gracias a los cuales fue un éxito.

\section{Referencias}

1. Abenza, L., Alarcón, F., Piñar, M.I. y Ureña, N. (2009). Relationship between the anxiety and performance of a basketball team during competition. Revista de Psicología del Deporte, 18(3), 409-413.

2. Abid, K., Alí, M., Hermassi, S., Shephard, R.J. \& Tabka, Z. (2010). Effects of in-season short-term plyometric training program on leg power, jump- and sprint performance of soccer players. The Journal of Strength and Conditioning Research, 24(10), 2670-2676.

3. Alarcón, F., Cárdenas, D., Escobar, R., Piñar, M.I. y Torre, E. (2009). La participación del jugador de minibásket en situaciones reducidas de juego en competición. Revista de Psicología del Deporte, 18(3), 445-449.

4. Alarcón, F., Carreño, F., Piñar, M.I., Rodríguez, D. y Vegas, A. (2002). Posiciones y distancias de lanzamiento durante la competición en Minibasket. En A. Díaz, P.L. Rodríguez, y J.A. Moreno (Coord.), Actas del III Congreso Internacional de Educación Física e Interculturalidad. Murcia: Consejería de Educación y Cultura de la Región de Murcia.

5. Almagro, B.J., Rebollo, J.A. y Sáenz-López, P. y Vizcaíno, C. (2013). Coaches' and referees' evaluation of the importance of the rules at the initiation stage of basketball. Revista de Psicologia del Deporte, 22(1), 293-297.

6. Altman, D. G. (1991). Practical Statistics for Medical Research. London: Chapman \& Hal.

7. American Academy of Pediatrics, Committee on Sports Medicine and Fitness. (2000). Risk of injury from baseball and softball in children. Pediatrics, 107(4), 782-784.

8. American Academy of Pediatrics. Committee on Sports Medicine and Fitness and Committee on School Health. (2001). Organized sports for children and preadolescents. Pediatrics, 107(6), 1459-1462.American Sport Education Program. (2001). Coaching youth basketball. Champaing, IL: Human Kinectics.

9. Anguera, M.T. (1986). La Investigación Cualitativa. Educar, 10, 23-50.

10. Anguera, M.T. (1995). Método de investigación en psicología. Madrid: Editorial Síntesis.

11. Anguera, M.T. y Blanco, A. (2003). Calidad de los datos registrados en el ámbito deportivo. En A. Hernández-Mendo: Psicología del Deporte 
(Vol. 2), Metodología (pp. 35-73). Buenos Aires: Tulio Guterman. Recuperado de http://www.efdeportes.com

12. Anguera, M.T., Pérez, F. y Valera, S. (2011). Un nuevo instrumento para la identificación de patrones de ocupación espacial. Psicothema, 23(4), 858-863.

13. Anguera, M.T. y Hernández-Mendo, A. (2013). La metodología observaciones en el ámbito del deporte. Revista de Ciencias del Deporte, 9(3), $135-160$.

14. Árias, J.L. (2007). Análisis de la zona de lanzamiento según el diseño de la línea de tres puntos en minibásket femenino. IV Congreso Ibérico de Baloncesto, 2-12.

15. Arias, J.L., Argudo, F.M. y Alonso, J.I. (2008). Inclusión de la línea de tres puntos en minibasket. Revista Internacional de Ciencias del Deporte, 4(13), 54-68.

16. Árias, J.L. (2012). Free-throw accuracy and success as a function of ball weight in 9- to 11-year-old male players. Motriz, 18(2), 338-344.

17. Arias, J.L. (2012). Las oportunidades y el éxito en el lanzamiento en la situación de uno contra uno en minibásquet. Revista Internacional de Medicina y Ciencias de la Actividad Física y el Deporte, 12(45), 23-34.

18. Árias, J.L., Alonso, J.I. y Yuste, J.L. (2012). Propiedades psicométricas y resultados de la aplicación de la escala de disfrute y competencia percibida en baloncesto de iniciación, Universitas Psychologica, 3(12), 975-956.

19. Arias, J.L.; Argudo, F.M. y Alonso, J.I. (2008). La situación de uno contra uno en minibásket femenino: análisis comparativo entre dos ubicaciones diferentes de la línea de tres puntos. Motricidad. European Journal of Human Movement, (20), 1-12.

20. Árias, J.L., Argudo, F.M. y Alonso, J.I. (2008). La inclusión de la línea de tres puntos en minibásket. Revista Internacional de Ciencias del Deporte. 4(13), 54-68.

21. Arias, J.L.; Argudo, F.M. y Alonso, J.I. (2009). Método objetivo para analizar dos modelos de la línea de tres puntos en minibasket. Revista Internacional de Medicina y Ciencias de la Actividad Física y el Deporte, 9(36), 349-365.

22. Árias, J.L.; Argudo, F.M. y Alonso, J.I. (2011). Las reglas como variables didácticas. Ejemplo en baloncesto de formación. Revista Internacional de Medicina y Ciencias de la Actividad Física y el Deporte, 11(43), 491-512.

23. Arias, J. L., Argudo, F. M., y Alonso, J. I. (2011). Effect of two different forms of three-point line on game actions in girls mini-basketball. South African Journal for Research in Sport Physical Education and Recreation, 33(1), 9-22.

24. Arias, J.L., Argudo, F.M. y Alonso, J.I. (2011). Las reglas como variables didácticas. Ejemplo en baloncesto de formación. Revista Internacional de Medicina y Ciencias de la Actividad Física y el Deporte, 11(43), 491-512.

25. Arias, J. L., Argudo, F. M., y Alonso, J. I. (2012). Distances and shooting zones as a function of mass of basketball among 9 to 11 years old male players. South African Journal for Research in Sport Physical Education and Recreation, 34(1), 1-11.

26. Arias, J. L., Argudo, F. M., y Alonso, J. I. (2012). Effect of Basketball Mass on Shot Performance Among 9-11 Year-Old Male Players. International Journal of Sports Science and Coaching, 7(1), 69-79.

27. Alarcón, F., Cárdenas, D., Escobar, R., Piñar, M.I., y Torre, E. (2009). Participation of minibasketball players during small-sided competitions. Revista de Psicología del Deporte, 18(3), 445-449.

28. Altman, D.G. (1991). Practical statistics for medical research. London: Chapman and Hall.

29. Baker, J. y Côté, J. (2003). Sport-Specific Practice and the Development of Expert Decision-Making in Team Ball Sports. Journal of Applied Sport Psychology, 15(1), 12-25.

30. Baquero, C., Cárdenas, D., y Piñar, M.I. (2001). Minibásket: ¿̨un deporte adaptado a los niños?. Clinic: Revista técnica de baloncesto, 55, 4-11.

31. Bazaco, M.J., Gómez, M.A., Ortega, E. y Salado, J. (2011). Opinión de los entrenadores sobre distribución de contenidos técnico-tácticos y pedagógicos en distintas categorías de baloncesto de formación. Cuadernos de Psicología del Deporte, 11(2), 51-62.

32. Buendía, L., Colas, P. y Hernández, F. (1998). Métodos de Investigación en Psicopedagogía. Madrid. McGraw-Hill.

33. Buttifant, D. y Hrysomallis, C. (2012). Influence of training years on upper-body strength and power changes during the competitive season for profesional Australian rules football players. Journal of Science and Medicine in Sport, 15(4), 374-378.

34. Blázquez, D. (1995). La iniciación deportiva y el deporte escolar. Barcelona: INDE.

35. Calvo, R.M., Lozano, C. y Ureńa, A. (2002). Estudio de la recepción del saque en el voleibol masculino español de elite tras la incorporación del jugador líbero. Revista Internacional de Medicina y Ciencias de la Actividad Fisica y el Deporte, 2(4), 37-49.

36. Cañadas, M., Feu, S., García, J., Ibáńez, S.J. y Parejo, I. y. (2013). Las situaciones de juego en el entrenamiento de baloncesto en categorías base-Game situations in youth basketball practices. Revista Internacional de Medicina y Ciencias de la Actividad Física y el Deporte, 13(45), 41-54.

37. Cárdenas, D. (2003). El proceso de formación táctica colectiva desde un punto de vista constructivista. En A. López, C. Jiménez y R. Aguado (Eds.). Didáctica del baloncesto en las etapas de formación, 179-209. Madrid: Editores.

38. Cárdenas, D. (2006). El proceso de formación táctica colectiva en el baloncesto desde la perspectiva constructivista. Lecturas: Educación Física y Deportes, (94), 33 .

39. Cárdenas, D. (2010). Fundamentos de las habilidades de los deportes de equipo: Baloncesto. Granada: Reprografía Digital.

40. Cárdenas, D., García, E. y Torre. E. (2001). La motivación en la prác tica físico-deportiva. Lecturas: Educación Física y Deportes, 39. Extraído de http://www.efdeportes.com/efd39/ motiv1.htm.

41. Cárdenas, D., Miranda, M.T., Piñar, M.I. y Torre, E. (2008). Factores que afectan al aprendizaje durante la competición e influyen en la formación del jugador de minibásket. Habilidad motriz: Revista de Ciencias de la Actividad Física y del Deporte, (31), 5-15.

42. Cárdenas, D. y Pintor, D. (2001). La iniciación al baloncesto en el medio escolar. En F. Ruiz, A. García y A. Casimiro, La iniciación deportiva basada en los deportes colectivos. (pp. 105-144). Madrid: Gymnos.

43. Cardon, G., Carreiro da Costa, F., De Bourdeaudhuij, I., Haerens, L. \& Van Acker, R. (2010). Sex equity and physical activity levels in coeducational physical education: exploring the potential of modified game forms. Physical Education and Sport Pedagogy, 15(2), 159-173.

44. Carrillo, A. y Rodríguez, J. (2009). El básquet a su medida. Pre-mini de 8 a 10 años. Zaragoza: INDE.

45. Casamichana, D., Castellano, J. y Delial, A. (2013). Influence of different training regimes on physical and physiological demands during small-sided soccer games: Continuous vs. Intermittent format. Journal of Strength and Conditioning Research, 27(3), 690-697.

46. Casey, A., Méndez, A. y Valero, A. (2010). What are we being told about how to teach games? A three-dimensional analysis of comparative research into different instructional studies in Physical Education and School Sports. Revista Internacional de Ciencias del Deporte, 6(18), 37-56.

47. Castro, J. (2004). Desarrollo de la noción de espacio en el nińo de Educación Inicial. Acción pedagógica, 13(2), 162-170.

48. Chalip, L. y Green, B.C. (1998). Sport tourism as the celebration of subculture. Annals of Tourism Research. Pergamon, 25(2). 275-291

49. Chase, M. A., Ewing, M. E., George, T. R. \& Lirgg, C. D., (1994). The effects of equipment modification on children's self-efficacy and basketball shooting performance. Research Quarterly for Exercise and Sport, 65(2), 159-168.

50. Corvo, A., Dwyer, D. B., Gabbett, T. J., Kearney, S., \& Polley, C. (2014). Influence of field position and phase of play on the physical 
demands of match-play in professional rugby league forwards. Journal of Science and Medicine in Sport, 17(5), 556-561.

51. Coulter, J. (1971). Decontextualized meaning: current approach to verstehen investigations. Sociological Review, 19(3), 301-326.

52. Dowling, S., Hassan, D., McConkey, R. \& Menke, S. (2012). Promoting social inclusion through unified sports for youth with intellectual disabilities: a five-nation study. Journal of Intellectual Disability Research, 57(10), 923-35.

53. Escudero, P. y Lasierra, G. (1993). Observación y evaluación en los deportes de cooperación-oposición: en busca de sus aspectos distintivos. Apunts, (31), 86-105.

54. Federación Andaluza de Baloncesto (2001). Reglamento de minibasket. Sevilla: FAB.

55. Federación Espańola de Baloncesto. Bases de Competición. Campeonato de Espańa de Selecciones Autonómicas de Minibásket. Temporada 2012-2013. Edición del 2 de Junio.

56. Federación Espańola de Baloncesto. Bases de competición. Campeonato de España de selecciones autonómicas de minibásket. Temporada 20132014. Edición 8 de Junio.

57. Federación de Baloncesto de la Región de Murcia. (2013). Reglamento de Minibasket. Bases de competición temporada 2013-2014. Murcia: FBRM.

58. García, E., Pérez, J.J. y Rodríguez, P.L. (2013). Autopercepción de competencia motriz, práctica físico-deportiva federada y su relación con los niveles de actividad física habitual en escolares. Revista Digital de Educación Física, 4(20). Recuperado de http://emasf.webcindario.com

59. García, E., Rodríguez, P.L., Pérez, J.J., Moral, J.E., López, P.A. y López, F.J. (2013). Autopercepción de competencia motriz y práctica físicodeportiva en alumnos de 10 a 12 ańos de Molina de Segura (MurciaEspaña). Acción Motriz, 11, 47-58.

60. Garganta, J. (2009). Trends of tactical performance analysis in team sports: bridging the gap between research, training and competition. Tactical analysis in team sports. Revista Portuguesa de Ciências do desporto, 9(1), 81-89.

61. Giménez, F.J. y Sáenz-López, P. (1996). La competición en la iniciación al baloncesto. Apunts: Educación Física y Deportes, (46), 42-52.

62. Giménez, J. y Sáenz-López, P. (1999). Principios metodológicos en E.F. En Sáenz-López, P.; Tierra, J. y Díaz Z, M. (coord.). Actas del XVII Congreso Nacional de E.F. Instituto Andaluz del Deporte. Málaga.

63. Gould, D. (1987). Understanding attrition in children's sport. En Gould, D. y Weiss, M.R. (Eds.). Advances in pediatric sport sciences. (6186) Champaign, Illinois: Human Kinetics.

64. Gómez, M.A., Ibáńez, S.J., Ortega, E. y Sampaio, J. (2008). Game location influences basketball players' performance across playing positions. International Journal of Sport Psychology, 39(3), 205-216.

65. Graça, A. y Oliveira, J. (1997). La enseñanza de los juegos deportivos. Barcelona: Paidotribo.

66. Graça, A. (1998). Comparing the high and the low achievers'opportunity to participate in basketball game within Physical Education classes. In M. Hughes \& F. Tavares (Eds.), IV World Congress of Notational Analysis of Sport (pp. 127-134). Porto: FCDEF-UP.

67. Hernández, Fernández y Baptista (2000). Metodología de la investigación. México: Mx Graw Hill.

68. Holt, N.L., Stream, W.B. y García, E. (2002). Expanding the Teaching Games for Understanding Model: New Avenues for Future Research and Practice, Journal of teaching in Physical Education, 21(2), pp. 162176.

69. Jiménez, J.M., Ortega, E., Palao, J.M. y Sainz de Baranda, P. (2008). Diseño y validación de un cuestionario para validar las preferencias y satisfacciones en jóvenes jugadores de baloncesto. Cuadernos de Psicologia del Deporte, 8(2), 39-58.

70. Kew, F. (1990). The development of games: an endogenous explanation. International Review for the Sociology of Sport, 25(4), 251-267.
71. Köklü, Y. et al. (2011). Comparison of the physiological responses to different small-sided games in elite young soccer players. Journal of Strength and Conditioning Research, 25(6), 1522-1528.

72. Lasierra, G. y Lavega, P. (1993): 1015 juegos y formas jugadas de iniciación a los deportes de equipo. Barcelona: Paidotribo.

73. Martín, C. (2006). La formación del educador deportivo en baloncesto. Bloque común. Sevilla: Wanceulen Editorial Deportiva, S.L.

74. Méndez, A. (1998). Los juegos de predominio táctico: una propuesta eficaz para la enseńanza de los deportes de invasión" en revista digital Lecturas de E. F. y Deportes, 11. Recuperado de http://www.efdeportes. com/efd11a/jtact.htm

75. Mignorance, A.C. y Torres, C. 1. (2006). La formación de educador deportivo en baloncesto. Bloque específico Nivel I. Sevilla: Wanceulen Editorial Deportiva, S.L 56

76. Mitjana, J. C. (2007). Propuesta de un nuevo reglamento de minibasket. Clinic: Revista Técnica de Baloncesto, 20(76), 36-41.

77. Mondoni, M. (2001). ¿¿Por qué en el minibasket es mejor la defensa individual?. Clinic, (52), 8-11.

78. Naranjo, M.C., Pajón, M.A. y Quintero, S. (2010). La preparación física y el minibásket. Wanceulen E.F. Digital, (7), 144-169.

79. Nathan et al. (2010). Social cohesion through football: a quasi-experimental mixed methods design to evaluate a complex health promotion program. Biomedcentral Public Health, 10(1), 587.

80. Navarro (2002). El afán de jugar. Teoría práctica de los juegos motores. Barcelona: Ed. INDE

81. Ortega, E. (2006). La competición como medio formativo en el baloncesto. Sevilla: Wanceulen.

82. Ortega, E. (2010). Medios técnico-tácticos colectivos en baloncesto en categorías de formación. Revista Internacional de Medicina y Ciencias de la Actividad Física y el Deporte, 10 (38), 234-244.

83. Ortega, E., Ortega, V., Palao, J. M. y Piñar, M. I. (2004). Incidencia de la inclusión de la línea de 3 puntos sobre el tanteo en baloncesto y propuesta de modificación en categorías de formación. Revista Digital: RendimientoDeportivo.com. Recuperado de http://www.rendimientodeportivo.com/N007/Artic035.htm.

84. Ortega, E. Piñar, M.I. y Cárdenas, D. (1999). El estilo de juego de los equipo de baloncesto en las etapas de formación. Granada: Ortega, Piñar y Cárdenas.

85. Ortega, E., Pińar, M. I., Salado, J., Palao, J. M. y Gómez, M. A. (2012). Opinión de expertos y entrenadores sobre el reglamento de la competición infantil en baloncesto. Revista Internacional de Ciencias del Deporte, 28(8), 142-150

86. Parlebas, P. (2001). Juegos, deportes y sociedad. Léxico de la praxiología motriz. Barcelona: Paidotribo.

87. Passos, P., Milho, J., Fonseca, S., Borges, J., Araújo, D. y Davids, K. (2011). Interpersonal Distance Regulates Functional Grouping Tendencies of Agents in Team Sports. Journal of Motor Behavior, 43(2), 155-163.

88. Perea-Milla, E. (1998). Estudios cuasi-experimentales y estudios aleatorios controlados. En R. Burgos (Eds), Metodología de investigación y escritura cientifica en clinica (pp. 147-176).Granada: Escuela Andaluza de Salud Pública.

89. Pintor, D. (1987). Apuntes de la asignatura de Baloncesto II. FCCAFD. Universidad de Granada. Inéditos.

90. Pinto, D., Graça, A. e Ibáńez, S. J. (2002). Operacionalizaçăo da avaliação dos alunos no jogo reduzido $3 \times 3$, em basquetebol. En $9^{\circ}$ Congresso de Educação Física e Ciências do Desporto dos Países de Lingua Portuguesa. Cultura e Contemporaneidade na Educação Fisica e no Desporto. E Agora? (pp. 34). Sao Luis: Universidade Federal do Maranhão \& Associação Prata da Casa.

91. Piñar, M. I. (2005). Incidencia en el cambio de un conjunto de reglas de juego sobre algunas variables que determinan el proceso de formación de los jugadores de minibasket 9-11 años. Tesis doctoral. Universidad de Granada. 
92. Rebollo, J.A., Sáenz-López, P. y Vizcaino, C. (2013). Revisión de los reglamentos de minibásket en las Comunidades Autónomas de España. Revista de Ciencias del Deporte, 9(3), 173-192.

93. Roberts, G. C. y Balagué, G. (1991). The development and validation of the perception of Success Questionnaire. Colonia, Alemania: Congress FEPSAC.

94. Rojas, F.J., Cepero M., Ońa, A. y Gutiérrez, M. (2000) Kinematic ad- justements in the basketball jump shot against an opponent. Ergonomics, 43(10), 1651-1660.

95. Sáez, G. (2008). Los valores a desarrollar a través de la enseñanza del baloncesto de iniciación. International Journal of Sports Law \& Management, (2), 32-46.

96. Sierra-Bravo R. (1995). Técnicas de investigación Social Teoría y ejercicios (10 a ed.). Madrid: Paraninfo. 
